\title{
Hole dynamics in canted antiferromagnets: Coexistence of many-body and free-like excitations
}

\author{
I. J. Hamad, ${ }^{1}$ L. O. Manuel, ${ }^{1}$ G. Martinez, ${ }^{2}$ and A. E. Trumper ${ }^{1}$ \\ ${ }^{1}$ Instituto de Física Rosario (CONICET) and Universidad Nacional de Rosario, Boulevard 27 de Febrero 210 bis, \\ (2000) Rosario, Argentina \\ ${ }^{2}$ Instituto de Física, UFRGS, 91501-970 Porto Alegre, RS, Brasil
}

(Received 2 June 2006; revised manuscript received 28 July 2006; published 15 September 2006)

\begin{abstract}
We have analyzed the dynamics of a single hole doped in a canted antiferromagnet using the $t$ - $J$ model. Within the self-consistent Born approximation we have found that the hole propagates at two different energy scales along the antiferromagnetic and the ferromagnetic components of the canted order, respectively. While the many body quasiparticle excitation has its origin in the coherent coupling of the hole with the magnon excitations of the antiferromagnetic component, the ferromagnetic component gives rise to a free-like hole motion at higher energies. We have found a nontrivial behavior of the hole spectral function with the canting angle $\theta$. In particular, in the strong coupling regime, the quasiparticle weight strongly depends on the momenta, vanishing inside the magnetic Brillouin zone for $\theta \gtrsim 60^{\circ}$.
\end{abstract}

DOI: 10.1103/PhysRevB.74.094417

PACS number(s): 75.50.Ee, 71.10.Fd

\section{INTRODUCTION}

The underlying physics behind the doped Mott insulators is essential to elucidate the mechanism that leads to the high temperature superconductivity. The major challenge is to explain, microscopically, how an antiferromagnetic (AF) insulator evolves into a superconductor. ${ }^{1}$ In this sense, angle resolved photoemission spectroscopy (ARPES) is one of the most refined experiments that gives access to the one particle excitations of doped Mott insulators. ${ }^{2}$ Although the photoemission spectra of the cuprates have been well characterized by ARPES experiments as a function of doping, there is still no consensus about the theoretical description of the multiple features observed. Even the most simple case, that is, the dynamics of a hole injected in an antiferromagnetic matrix, remains still a controversial issue. In particular, the main disagreement is that in most of the theoretical calculationsbased on the $t-J$ model-the low energy excitations corresponds to quasiparticle excitations, ${ }^{3}$ while in the ARPES spectra the width of the low energy peaks is so large that they cannot be associated with a physical lifetime of quasiparticles. ${ }^{2,4} \mathrm{~A}$ first proposal to resolve such a disagreement invoked the spin-charge separation scenario, ${ }^{5}$ although several recent works ${ }^{6}$ have pointed out that the electronphonon coupling can lead to the observed overdamped quasiparticle excitations. In addition to this controversy observed in the cuprates, there has been an increasing interest in the study of the hole motion in different antiferromagnetic backgrounds in order to test the validity of the coherent quasiparticle-or spin polaron-picture. For the unfrustrated $180^{\circ}$ Néel order it has been shown, numerically and analytically, ${ }^{3,7-11}$ that the quasiparticle excitations exist for all momenta and for all $J>0$. On the other hand, in a highly frustrated case such as the kagomé lattice, which is believed to be magnetically disordered, completely incoherent spectral functions have been found ${ }^{12}$ for all momenta, $J / t=0.4$, and both $t$ signs - it should be noted that in the square lattice unfrustrated case the particle-hole symmetry leads to the same behavior for both signs of $t$. In addition, we have recently found ${ }^{13}$ that in the triangular antiferromagnet, with a $120^{\circ}$ Néel order, an intermediate situation between the square and the kagomé geometries arises, since the quasiparticle weight only vanishes for $t>0$. This result is particularly interesting because it means that the conventional quasiparticle picture can be broken in a semiclassical magnetic background, without invoking spin liquid phases. In addition to the mechanism of hole motion assisted by spin fluctuations, already existing in the unfrustrated case, in the triangular antiferromagnet there appears a free hopping hole mechanism as a direct consequence of a ferromagnetic component of the underlying magnetic structure. The latter implies a finite probability of hole motion without emission or absorption of magnons. In our previous work we have shown that it is the subtle interference between both processes for hole motion that produces the vanishing of the quasiparticle excitations. ${ }^{13}$

In the present article we will study the hole dynamics in canted antiferromagnetic states where, by varying the canting angle, it is possible to consider the evolution of the magnetic background from the antiferromagnetic to the ferromagnetic state. This kind of study, little explored in the literature, ${ }^{14}$ allows us to investigate the spectral functions continually from the AF state-only spin flip assisted processes - to the ferromagnetic state-only free hopping process-so as to investigate more carefully how the nontrivial interference between both hole-motion processes influences the formation of a coherent quasiparticle. To carry out the study we have derived an effective Hamiltonian from the $t-J$ model using the spinless fermion representation for the kinetic part, and a canted spin wave state for the magnetic part. Within the self-consistent Born approximation we have found that, for $J<t$, the hole propagates preferably at two well separated energies: as a coherent spin polaron excitation at low energy, and as a quasifree hole at higher energy. We were able to associate the former energy scale to the AF component, while the latter to the ferromagnetic one. As the canting angle increases we have observed an important spectral weight transfer from low to higher energy sectors leading to the reduction of the quasiparticle weight. In particular, inside the magnetic Brillouin zone (MBZ), the quasiparticle weight vanishes for $\theta \gtrsim 60^{\circ}$. 
This article is organized as follows. In Sec. II we formulate the effective Hamiltonian for a hole moving in a canted AF. In Sec. III we briefly draw the calculation of the hole Green function within the self-consistent Born approximation. In Sec. IV we present and discuss the results for the hole spectral functions, and in Sec. V we state the conclusions of our work.

\section{EFFECTIVE HAMILTONIAN FOR A HOLE IN A CANTED ANTIFERROMAGNET}

To study the hole motion in a canted antiferromagnet we will study the $t-J$ model in the square lattice. ${ }^{9}$ In order to stabilize a canted phase we add a Zeeman term that couples only with the spin operators, so that we can vary the canting angle $\theta$ by tuning a fictitious uniform magnetic field $B$. Thus, we use the following $t-J$ Hamiltonian:

$$
H=H_{t}+H_{J}=-t \sum_{\langle i, j\rangle}\left(\hat{c}_{i, \sigma}^{\dagger} \hat{c}_{j, \sigma}+\mathrm{H} . \mathrm{c} .\right)+J \sum_{\langle i, j\rangle} \mathbf{S}_{i} \cdot \mathbf{S}_{j}+B \sum_{i} S_{i}^{z},
$$

where the kinetic part $H_{t}$ represents the hopping between nearest neighbors $(\langle i, j\rangle)$ of the square lattice, with the constraint of no double occupancy $\hat{c}_{i, \sigma}=c_{i, \sigma}\left(1-n_{i,-\sigma}\right)$ and $H_{J}$ represents the AF Heisenberg part along with the Zeeman term.

It should be stressed that the realization of canted phases-stabilized in our study by a fictitious magnetic field-actually can be a consequence of the interplay of Dzyaloshinskii-Moriya and further anisotropy terms, such as those that are responsible for the weak ferromagnetism in $\mathrm{La}_{2} \mathrm{CuO}_{4}$. Furthermore, a similar effective Hamiltonian, as the one we will obtain below [see Eq. (8)], can be derived in the pure $t-J$ model with additional hopping terms to second and third neighbors. The latter model may be relevant to describe recent ARPES spectra of $\mathrm{Ca}_{2} \mathrm{CuO}_{2} \mathrm{Cl}_{2} \cdot{ }^{15} \mathrm{So}$, the hole dynamics in both cases-canted and further hoppings $t-J$ models — will be closely related.

\section{A. Magnetic part}

The magnetic part is treated in the spin wave approximation. ${ }^{16}$ It is assumed that the semiclassical uncanted AF order lies in the $x-y$ plane while the magnetic field $B$ points in the $z$ direction. So, the effect of the magnetic field is to tilt the spins an angle $\theta$ out of the $x-y$ plane. The generalized Holstein-Primakov transformation for the canted case results

$$
\begin{gathered}
\mathbf{S}_{i}^{x}=\cos \theta\left(S-a_{i}^{\dagger} a_{i}\right)-i \sqrt{S / 2} \sin \theta\left(a_{i}-a_{i}^{\dagger}\right), \\
\mathbf{S}_{i}^{y}=\sqrt{S / 2}\left(a_{i}+a_{i}^{\dagger}\right), \\
\mathbf{S}_{i}^{z}=-\sin \theta\left(S-a_{i}^{\dagger} a_{i}\right)-1 \sqrt{S / 2} \cos \theta\left(a_{i}-a_{i}^{\dagger}\right),
\end{gathered}
$$

for $i \in$ sublattice $A$, and

$$
\begin{gathered}
\mathbf{S}_{j}^{x}=-\cos \theta\left(S-b_{j}^{\dagger} b_{j}\right)+1 \sqrt{S / 2} \sin \theta\left(b_{j}-b_{j}^{\dagger}\right), \\
\mathbf{S}_{j}^{y}=\sqrt{S / 2}\left(b_{j}+b_{j}^{\dagger}\right),
\end{gathered}
$$

$$
\mathbf{S}_{j}^{z}=\sin \theta\left(S-b_{j}^{\dagger} b_{j}\right)+1 \sqrt{S / 2} \cos \theta\left(b_{j}-b_{j}^{\dagger}\right),
$$

for $j \in$ sublattice $B$. The Fourier transform of the bosonic operators $a$ 's and $b$ 's are defined as

$$
a_{i}^{\dagger}=\sqrt{\frac{2}{N}} \sum_{\mathbf{k}} e^{\mathbf{k} R_{i}} a_{\mathbf{k}}^{\dagger}, \quad b_{j}^{\dagger}=\sqrt{\frac{2}{N}} \sum_{\mathbf{k}} e^{\mathbf{k} R_{j}} b_{\mathbf{k}}^{\dagger},
$$

where $N$ is the number of lattice sites and $\mathbf{k}$ runs along the magnetic Brillouin zone (half of the square lattice Brillouin zone). Up to quadratic order, the magnetic part of the $t-J$ Hamiltonian takes the form

$$
\begin{aligned}
H_{J}= & E_{C}+\sum_{\mathbf{k}}\left[A\left(a_{\mathbf{k}}^{\dagger} a_{\mathbf{k}}+b_{\mathbf{k}}^{\dagger} b_{\mathbf{k}}\right)+D \gamma_{\mathbf{k}}\left(a_{\mathbf{k}}^{\dagger} b_{\mathbf{k}}+a_{\mathbf{k}} b_{\mathbf{k}}^{\dagger}\right)\right. \\
& \left.+C \gamma_{\mathbf{k}}\left(a_{\mathbf{k}} b_{-\mathbf{k}}+a_{\mathbf{k}}^{\dagger} b_{-\mathbf{k}}^{\dagger}\right)\right]
\end{aligned}
$$

with $A=4 J S \cos 2 \theta+B \sin \theta, D=J S \sin ^{2} \theta, C=J S \cos ^{2} \theta$, and

$$
E_{c}=-2 J S^{2} N \cos ^{2} \theta-B N S \sin \theta .
$$

Minimization of $E_{c}$ with respect to $\theta$ leads to $B=8 J S \sin \theta$. This value cancels the linear bosonic terms of the magnetic part and justify the use of the quadratic form (2). Furthermore, if this value of $B$ is replaced in $E_{c}$ and $A, H_{J}$ can be written as function of the canting angle $\theta$ only. If $H_{J}$ is expressed in a matrix form the dynamical matrix has a dimension $4 \times 4$. The diagonalization of $H_{J}$ is performed in two steps: first, a transformation $\alpha_{\mathbf{k}}^{\dagger}=a_{\mathbf{k}}^{\dagger}+b_{\mathbf{k}}^{\dagger}$ and $\beta_{\mathbf{k}}^{\dagger}=a_{\mathbf{k}}^{\dagger}-b_{\mathbf{k}}^{\dagger}$ that renders the dynamical matrix diagonal by blocks of $2 \times 2$ and, then, a Bogoliubov transformation from $\alpha, \beta$ to the new bosonic operators $\eta, \nu$

$$
\begin{gathered}
\alpha_{\mathbf{k}}=u_{\mathbf{k}}^{+} \eta_{\mathbf{k}}+v_{\mathbf{k}}^{+} \eta_{-\mathbf{k}}^{\dagger}, \\
\beta_{\mathbf{k}}=u_{\mathbf{k}}^{-} \nu_{\mathbf{k}}+v_{\mathbf{k}}^{-} \nu_{-\mathbf{k}}^{\dagger}
\end{gathered}
$$

with Bogoliubov coefficients

$$
u_{\mathbf{k}}^{ \pm}=\left(\frac{A \pm D \gamma_{\mathbf{k}}}{2 \omega_{\mathbf{k}}^{ \pm}}+\frac{1}{2}\right)^{1 / 2}
$$

and

$$
v_{\mathbf{k}}^{ \pm}=\mp \frac{\gamma_{\mathbf{k}}}{\left|\gamma_{\mathbf{k}}\right|}\left(\frac{A \pm D \gamma_{\mathbf{k}}}{2 \omega_{\mathbf{k}}^{ \pm}}-\frac{1}{2}\right)^{1 / 2} .
$$

Once these transformations are taken into account the harmonic magnetic part results

$$
H_{J}=E_{c}-2 J S N+\sum_{\mathbf{k}}\left[\left(\omega_{\mathbf{k}}^{+}+\omega_{\mathbf{k}}^{-}\right)+\omega_{\mathbf{k}}^{+} \eta_{\mathbf{k}}^{\dagger} \eta_{\mathbf{k}}+\omega_{\mathbf{k}}^{-} \nu_{\mathbf{k}}^{\dagger} \nu_{\mathbf{k}}\right]
$$

with the two magnon dispersion branches

$$
\omega_{\mathbf{k}}^{ \pm}=2 J S \sqrt{\left(1 \pm \gamma_{\mathbf{k}}\right)\left(1 \mp \cos \theta \gamma_{\mathbf{k}}\right)},
$$

defined in the magnetic Brillouin zone and $\gamma_{\mathrm{k}}=\left(\cos k_{x}\right.$ $\left.+\cos k_{y}\right) / 2$. As $\mathbf{k} \rightarrow(0,0)$, the dispersion $\omega_{\mathbf{k}}^{-} \rightarrow 0$ linearly, while $\omega_{\mathbf{k}}^{+} \rightarrow 4 J S \sin \theta$ quadratically. For this reason, hereafter, $\omega_{\mathbf{k}}^{-}$and $\omega_{\mathbf{k}}^{+}$will be called the $\mathrm{AF}$ and ferromagnetic bands, respectively. Furthermore, since $\omega_{\mathbf{k}+(\pi, \pi)}^{+}=\omega_{\mathbf{k}}^{-}$, it can be seen that in the one magnon operator description, where the translational symmetry of the square lattice is not broken, it is 
recovered the same dispersion of Ref. 17 if $\omega_{\mathbf{k}}^{+}$is unfolded to the square Brillouin zone.

\section{B. Kinetic part}

In order to fulfill the basic requirement of the $t-J$ model, the no double occupancy constraint, we use the following spinless fermion transformation ${ }^{3}$ for the canted case:

$$
\begin{aligned}
& \hat{c}_{i \uparrow}=\cos \frac{\theta}{2} g_{i}+\sin \frac{\theta}{2} g_{i}^{\dagger} a_{i}, \\
& \hat{c}_{i \downarrow}=\cos \frac{\theta}{2} g_{i}^{\dagger} a_{i}-\sin \frac{\theta}{2} g_{i},
\end{aligned}
$$

for $i \in$ sublattice $A$

$$
\begin{aligned}
& \hat{c}_{j \uparrow}=\sin \frac{\theta}{2} f_{j}+\cos \frac{\theta}{2} f_{j}^{\dagger} b_{j}, \\
& \hat{c}_{j \downarrow}=\sin \frac{\theta}{2} f_{j}^{\dagger} b_{j}-\cos \frac{\theta}{2} f_{j},
\end{aligned}
$$

for $j \in$ sublattice $B$, where $g_{i}, f_{j}$ are the fermionic hole operators and $a_{i}, b_{j}$ are the Holstein Primakov bosons. Replacing Eqs. (4) and (5) in the kinetic part of Eq. (1), and retaining terms up to third order it results

$$
\begin{aligned}
H_{t}= & -t \sin \theta \sum \gamma_{\mathbf{k}}\left(g_{\mathbf{k}} f_{\mathbf{k}}^{\dagger}+f_{\mathbf{k}} g_{\mathbf{k}}^{\dagger}\right) \\
& -t \sqrt{\frac{2}{N}} \cos \theta \sum_{\mathbf{k} \cdot \mathbf{k}^{\prime}}\left[\gamma_{\mathbf{k}} g_{\mathbf{k}} f_{\mathbf{k}^{\prime}}^{\dagger}, b_{\mathbf{k}^{\prime}-\mathbf{k}}\right. \\
& \left.-\gamma_{\mathbf{k}^{\prime}} g_{\mathbf{k}} f_{\mathbf{k}^{\prime}}^{\dagger} a_{\mathbf{k}-\mathbf{k}^{\prime}}^{\dagger}+\mathrm{H} . \mathrm{c} .\right] .
\end{aligned}
$$

Now, it is convenient to define the new fermionic bonding and antibonding operators

$$
g_{\mathbf{k}}=\frac{l_{\mathbf{k}}+m_{\mathbf{k}}}{\sqrt{2}}, \quad f_{\mathbf{k}}=\frac{l_{\mathbf{k}}-m_{\mathbf{k}}}{\sqrt{2}},
$$

respectively. When fermions $g$ and $f$ are expressed as a function of the bonding and antibonding fermions (7), and the bosons $a$ and $b$ in terms of the Bogoliubov operators $\eta, \nu$, the kinetic part (6) can be rewritten as

$$
\begin{aligned}
H_{t}= & \sum_{\mathbf{k}} \epsilon_{\mathbf{k}}\left(m_{\mathbf{k}}^{\dagger} m_{\mathbf{k}}-l_{\mathbf{k}}^{\dagger} l_{\mathbf{k}}\right)+\sqrt{\frac{2}{N}} \sum_{\mathbf{k} \cdot \mathbf{q}}\left\{\left[M_{\mathbf{q}, \mathbf{k}}^{+} \eta_{\mathbf{k}-\mathbf{q}}^{\dagger}\left(m_{\mathbf{k}} l_{\mathbf{q}}^{\dagger}-l_{\mathbf{k}} m_{\mathbf{q}}^{\dagger}\right)\right.\right. \\
& \left.+ \text { H.c. }]+\left[M_{\mathbf{q}, \mathbf{k}}^{-} \nu_{\mathbf{k}-\mathbf{q}}^{\dagger}\left(l_{\mathbf{k}} l_{\mathbf{q}}^{\dagger}-m_{\mathbf{k}} m_{\mathbf{q}}^{\dagger}\right)+\text { H.c. }\right]\right\},
\end{aligned}
$$

with the free hopping band $\epsilon_{\mathbf{k}}=4 t \sin \theta \gamma_{\mathbf{k}}$ and the vertex interactions

$$
M_{\mathbf{q}, \mathbf{k}}^{ \pm}=-\frac{t}{2} \cos \theta\left(u_{\mathbf{k}-\mathbf{q}}^{ \pm} \gamma_{\mathbf{q}}-v_{\mathbf{q}-\mathbf{k}}^{ \pm} \gamma_{\mathbf{k}}\right) .
$$

There are two mechanisms for charge motion. The first one is a free hopping process, first term of Eq. (8), that naturally appears with the canting of the AF order. The second one represents a hopping process magnon assisted by the ferromagnetic and the antiferromagnetic bands, respectively.

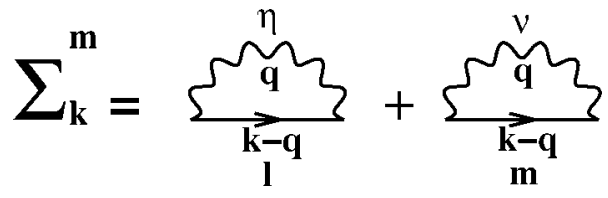

FIG. 1. Relevant contributions in the SCBA to the self-energy of the Green function corresponding to the antibonding fermion $m$. The wiggly lines represents free magnon Green functions for $\nu$ and $\eta$, while the straight lines represents the dressed fermionic Green functions $G^{m}$ and $G^{l}$. For the self-energy of the bonding fermion $l$, $m$, and $l$ must be interchanged.

When the canting angle $\theta=0^{\circ}$, the free hopping term vanishes and two degenerate antiferromagnetic bands are recovered. So, as it is expected for the case of hole motion in a pure AF matrix, the kinetic part is only described by a hole coupled to AF magnons. ${ }^{3,7}$ On the other hand, when the canting angle is such that the underlying magnetic order is ferromagnetic, $\theta=90^{\circ}$, the magnon assisted hopping disappears and the only mechanism available for hole motion is the free hopping term. Therefore, it is possible to interpolate the hole motion continually between the pure AF state and the ferromagnetic state, so as to investigate carefully how the nontrivial interference between both hole-motion processes influences the formation of a coherent quasiparticle.

\section{THE SELF-CONSISTENT BORN APPROXIMATION}

The use of the two magnetic sublattices requires the definition of the two Green functions [see Eq. (8)]

$$
G_{\mathbf{k}}^{m}(\omega)=\frac{1}{\omega-\epsilon_{\mathbf{k}}-\Sigma_{\mathbf{k}}^{m}(\omega)}, \quad G_{\mathbf{k}}^{l}(\omega)=\frac{1}{\omega+\epsilon_{\mathbf{k}}-\Sigma_{\mathbf{k}}^{l}(\omega)}
$$

along the magnetic Brillouin zone. Taking into account the interacting terms of the Hamiltonian (8) within the selfconsistent Born approximation, it is straightforward to see that there are two contributions to each self-energy (see, for instance, Fig. 1 for the self-energy of the antibonding fermion $m_{\mathbf{k}}$ ).

A standard procedure leads to two coupled self-consistent equations for the self-energies,

$$
\begin{aligned}
\sum_{\mathbf{k}}^{m(l)}(\omega)= & \sum_{\mathbf{q}}\left\{\left|M_{\mathbf{k}+\mathbf{q}, \mathbf{k}}^{+}\right|^{2} G_{\mathbf{k}+\mathbf{q}}^{l(m)}\left(\omega-\omega_{\mathbf{q}}^{+}\right)\right. \\
& \left.+\left|M_{\mathbf{k}+\mathbf{q}, \mathbf{k}}^{-}\right|^{2} G_{\mathbf{k}+\mathbf{q}}^{m(l)}\left(\omega-\omega_{\mathbf{q}}^{-}\right)\right\},
\end{aligned}
$$

that will be solved numerically. At this point, it is enlightening to relate the bonding $G_{\mathbf{k}}^{m}(\omega)$ and antibonding $G_{\mathbf{k}}^{l}(\omega)$, defined in the magnetic Brillouin zone, with the more physical hole Green function $G_{\mathbf{k}}^{h}(\omega)$ defined in the whole Brillouin zone. The hole operator $h$ is defined as $h_{i}=f_{i}$ for $i \in$ sublattice $A$ and $h_{i}=g_{i}$ for $i \in$ sublattice $B$. Then, if $h_{\mathbf{k}}$ is splitted as

$$
h_{\mathbf{k}}=\frac{1}{N} \sum_{i \in A} f_{i} e^{\mathbf{i k} \cdot \mathbf{R}_{i}}+\frac{1}{N} \sum_{i \in B} g_{i} e^{i \mathbf{k} \cdot \mathbf{R}_{i}},
$$

it is straightforward to find the operatorial relations $h_{\mathbf{k}}$ $=\frac{1}{\sqrt{2}}\left(f_{\mathbf{k}}+g_{\mathbf{k}}\right)=l_{\mathbf{k}}$ for $\mathbf{k}$ inside the magnetic Brillouin zone and 


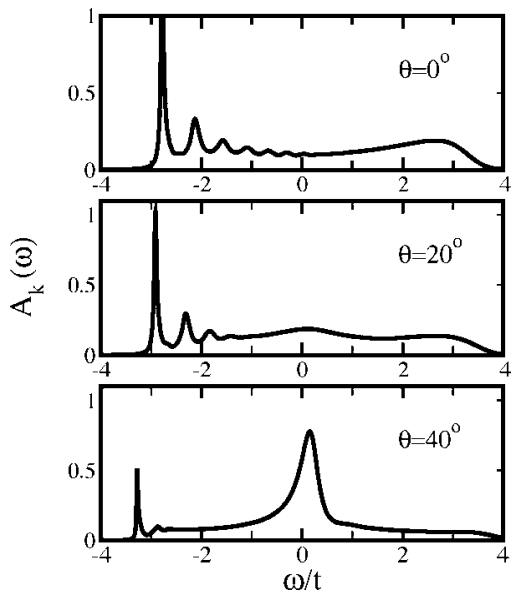

FIG. 2. Spectral function for $\mathbf{k}=(\pi / 2, \pi / 2)$ and $J / t=0.1$.

$h_{\mathbf{k}}=\frac{1}{\sqrt{2}}\left(f_{\mathbf{k}+\mathbf{Q}}-g_{\mathbf{k}+\mathbf{Q}}\right)=m_{\mathbf{k}+\mathbf{Q}}$ for $\mathbf{k}$ outside the magnetic Brillouin zone. These relations between the $h_{\mathbf{k}}, l_{\mathbf{k}}$, and $m_{\mathbf{k}}$, imply the following relation between the Green functions:

$$
\begin{gathered}
G_{\mathbf{k}}^{h}=G_{\mathbf{k}}^{l} \text { inside MBZ, } \\
G_{\mathbf{k}}^{h}=G_{\mathbf{k}+\mathbf{Q}}^{m} \text { outside MBZ. }
\end{gathered}
$$

\section{RESULTS}

\section{A. Spectral function}

We have numerically solved the self-consistent equation (10) for $\Sigma_{\mathbf{k}}^{m(l)}(\omega)$, using cluster sizes up to $N=40 \times 40$ and a frequency grid of 20000 points. Then, we calculated the corresponding spectral function $A_{\mathbf{k}}^{h}(\omega)=-\frac{1}{\pi} \operatorname{Im} G_{\mathbf{k}}^{h}(\omega)$ for several canting angles.

In Fig. 2 it is shown the spectra for $\mathbf{k}=(\pi / 2, \pi / 2)$ and $J / t=0.1$. We have chosen these particular momentum and coupling regime since for them, the main features of the spectra are clearly differentiated, and therefore it is easier to identify the underlying mechanisms for hole motion. The case $\theta=0^{\circ}$ (upper panel) corresponds to the spectral function of a hole in a pure AF matrix. This result has been already obtained by one of us ${ }^{7}$ and others authors ${ }^{3}$ using the SCBA. The spectra extend over a frequency range $\sim 8 t$ with a low energy sector composed by a delta peak at the bottom of the spectra along with several resonances of finite lifetime above it. The former is associated with a quasiparticle excitation (QP), whose bandwidth is of order $J$; whereas the latter resonances can be identified with string excitations since its energies scale as $E_{\text {string }} \sim(J / t)^{2 / 3}$. On the other hand, there is an incoherent part corresponding to the shoulder located at an energy $\sim 3 t$.

The low and high energy structure of the spectra can be traced back to the coupling between the hole and the underlying AF order. Low energy sector: as the hole moves, with a characteristic time of order $1 / t$, the AF order is locally disturbed leaving a string of overturned spins. Meanwhile, the zero point spin fluctuations above the classical Néel state $|N\rangle$, contained in the quantum AF ground state $|A F\rangle$ $=\exp \left(-\Sigma_{i j} u_{i j} a_{i}^{\dagger} b_{j}^{\dagger}\right)|N\rangle,{ }^{18}$ repair pairs of frustrated spins at a characteristic time of order $1 / J$. It is clear that, in the weak coupling regime $(J>t)$, the magnetic string of overturned spins can be completely erased by the zero point spin fluctuations, and the hole surrounded by an AF cloud emerges as a coherent quasiparticle excitation. However, in the strong coupling regime $(J<t)$, the magnetic string is only partially erased and, besides the low energy QP excitation, there are higher energy processes-strings excitations - corresponding to the hole inside a linear potential generated by the overturned spins. This picture has been widely confirmed by several numerical and analytical techniques. ${ }^{3,7,9-11}$ High energy sector: to describe the incoherent part of the high energy sector, it is convenient to take a closer look at the zero point spin fluctuations. In particular, if the exponential function in $|\mathrm{AF}\rangle$ is developed in a Taylor series, the quantum AF ground state can be written as $|\mathrm{AF}\rangle=|N\rangle+\mid$ fluct $\rangle$, where the fluctuations can be seen as a sum of $S^{z}$ conserving terms such as $a_{i_{1}}^{\dagger} a_{i_{2}}^{\dagger} \cdots a_{i_{n}}^{\dagger} b_{j_{1}}^{\dagger} b_{j_{2}}^{\dagger} \cdots b_{j_{n}}^{\dagger}|N\rangle$. As we have stated before, these fluctuations erase part of the strings, but they also generate small ferromagnetic clusters wherein the hole can propagate freely. This explains the broad shoulder centered at $\omega \sim 3 t$, that is, the finite probability to find the hole propagating at considerable high energies above the QP excitation. In fact, we have extended our calculation to the anisotropic Heisenberg model, and we have effectively found a suppression of the shoulder as well as an enhancement of the string resonances as the Ising limit is approached.

Now we discuss the evolution of the spectra with the canting angle. At $\theta=20^{\circ}$ (middle panel of Fig. 2), there appears a classical ferromagnetic component in the underlying magnetic order, while the zero point spin fluctuations get reduced. This adds a free hopping mechanism for the hole motion, represented by the tight binding term of Eq. (8). Such an additional mechanism competes with the magnonassisted, as well as with the incoherent hopping processes driven by the zero point spin fluctuations, resulting in a spectral weight transfer from the low energy sector and the incoherent shoulder to an energy located at $\omega \sim 0$. For $\theta=40^{\circ}$ (lower panel of Fig. 2) most of the spectral weight is dominated by this new mechanism, signaled by a finite lifetime resonance, $t$ resonance, located at an energy close to $\epsilon_{\mathrm{k}}=$ $-t \sin \theta \gamma_{\mathbf{k}}$. So that, as the canting angle increases, the probability of finding the hole moving freely along the classical ferromagnetic channel becomes more important than both, the magnon assisted and the incoherent hopping processes driven by zero point spin fluctuations. A similar scenario of a low energy excitation coexisting with a higher energy longlived resonance, dispersing as a free band, has been found in recent high-resolution photoemission spectra from the insulating cuprates $\mathrm{Ca}_{2} \mathrm{CuO}_{2} \mathrm{Cl}_{2} \cdot{ }^{15}$ While in the cuprate the appearance of the high energy part of the spectra has been ascribed to hoppings to first and further neighbors, in our present calculation the high energy part, dispersing as a free band, is obtained with the canting angle.

The evolution of the spectral function with $\theta$ becomes more complex when the $\mathbf{k}$ dependence is taken into account. In particular, for $\mathbf{k}$ outside the magnetic Brillouin zone the QP and string excitations start to overlap with the $t$ resonance 


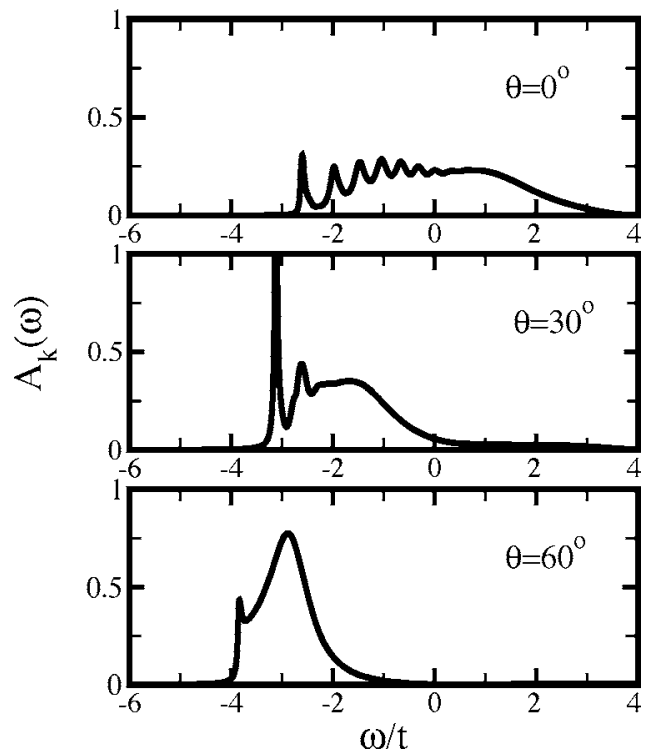

FIG. 3. Spectral function for $\mathbf{k}=(0.8 \pi, 0.8 \pi)$ and $J / t=0.1$.

at the low energy sector (see Fig. 3), while for $\mathbf{k}$ inside the magnetic Brillouin zone something similar to $\mathbf{k}$ $=(\pi / 2, \pi / 2)$ in Fig. 2 occurs, namely, the peaks are quite separated. As the canting angle is increased, there is a transfer of spectral weight from the magnon assisted to the free hopping process as a consequence of the reduction of the vertex interaction with $\theta, M_{\mathbf{q}, \mathbf{k}}^{ \pm} \sim \cos \theta$. It is worth stressing that the low energy QP excitation has its origin in the coherent scattering between the hole and the magnons, so the reduction of the scattering rate renders the magnon-assisted hopping process less effective than the free hopping one, that is originated by the ferromagnetic component of the underlying magnetic structure. Finally, for $\theta=90^{\circ}$, the only allowed hole motion process is the free one along the completely ferromagnetic order, thus, the whole spectral function becomes a single delta peak.

\section{B. Quasiparticle excitations}

In this section we analyze the quasiparticle excitations in the low energy sector of the spectra. This can be quantified by the QP weight $z_{\mathbf{k}}=\left|\left\langle\Phi_{\mathbf{k}}\left|h_{\mathbf{k}}^{\dagger}\right| \mathrm{AF}\right\rangle\right|^{2}$ that gives a measure of the overlap between the state of a bare hole created on the AF background and the quasiparticle state $\left|\Phi_{\mathbf{k}}\right\rangle$. In our case we computed the QP weight using the well known relation $z_{\mathbf{k}}=\left[1-\partial \Sigma_{\mathbf{k}}(\omega) /\left.\partial \omega\right|_{E_{\mathbf{k}}}\right]^{-1}$. It is worth to note that the complex $\mathbf{k}$ dependence of the spectral weight transfer with $\theta$, mentioned in the previous section, is clearly manifested in the QP excitations. In Fig. 4 it is shown the QP weight versus $\theta$ for $J / t=0.4$ and several momenta.

On one hand, for $\mathbf{k}$ inside the magnetic Brillouin zone the effect of the canting is to monotonically decrease the QP weight until it vanishes at around $\theta \sim 60^{\circ}$ [see, for instance, $\mathbf{k}=(\pi / 2, \pi / 2)$ and $\mathbf{k}=(0,0)$ of Fig. 4]. In this region the spectra is characterized by the two well separated peaks (see $\theta=40^{\circ}$ in Fig. 2) where the $\mathrm{QP}$ excitations have a magnon assisted origin. So, the vanishing of the QP weight with $\theta$ is

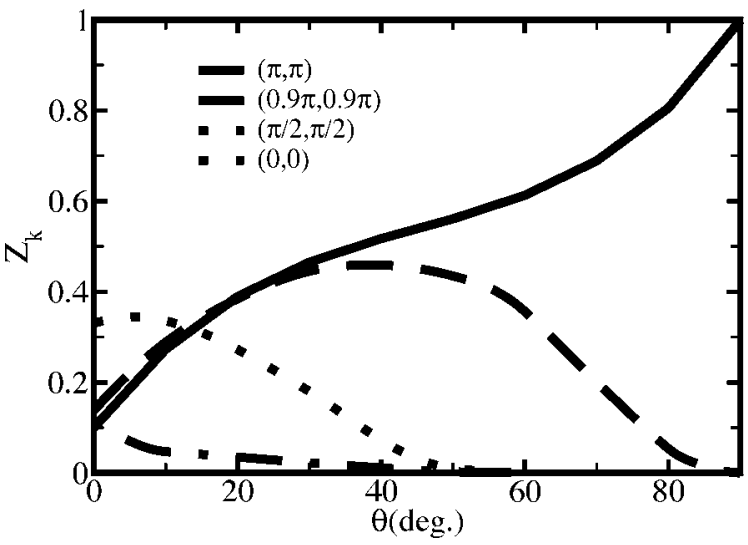

FIG. 4. QP weight as a function of the canting angle $\theta$ for several momenta and $J / t=0.4$.

due to the reduction of the vertex interaction with $\theta$. On the other hand, for $\mathbf{k}$ outside the magnetic Brillouin zone- $k$ $=(\pi, \pi)$ and $k=(0.8 \pi, 0.8 \pi)$-initially the QP weight increases with $\theta$. This is an unexpected behavior if the $\theta$ dependence of the vertex interaction is taken into account again. However, in this sector of the Brillouin zone, the magnon assisted and the free hopping processes merge at the low energy sector increasing the $\mathrm{QP}$ weight (see $\theta=30^{\circ}$ in Fig. $3)$. This can be seen as a constructive interference between the hole motion processes in the formation of the QP. For greater angles the QP weight is dominated by the vertex interaction and goes to zero as $\theta \rightarrow 90^{\circ}$. At $\theta=90^{\circ}$ the magnon assisted process vanishes for all $\mathbf{k}$ and the only allowed process is the free hole motion along the ferromagnetic channel, jumping $z_{\mathbf{k}}$ from zero to unity. $\mathbf{k}=(\pi, \pi)$ is a unique case where the QP weight increases monotonically to unity due to the strict energy coincidence of both hole motion processes. For this case we can say that there is always a constructive interference of the processes. It should be noted that the QP ground state momentum evolves with the canting angle along the diagonal $(\pi / 2, \pi / 2) \rightarrow(\pi, \pi),(\pi, \pi)$ being the ground state momentum for $\theta$ greater than $40^{\circ}$ when $\mathrm{J} / \mathrm{t}$ $=0.4$.

The advantage of using explicitly two sublattices in our calculation is the possibility to analyze separately the coupling of the hole with the ferromagnetic and AF magnons. For instance, if we cancel $M^{+}\left(M^{-}\right)$in the kinetic part (8), the coupling of the hole with the ferromagnetic (AF) band is omitted. Under this condition the self-consistent equations (10) for $\Sigma_{\mathbf{k}}^{m(l)}(\omega)$ can be solved and, via the relations (11), it is obtained $G_{\mathbf{k}}^{h}(\omega)$ without the effect of the ferromagnetic or the AF excitations on the hole motion.

For instance, in Fig. 5 it is shown separately the ferro (dashed line) and the AF (dotted line) contributions to the QP weight, along with the complete prediction (solid line), for $J / t=0.4$ and $\theta=40^{\circ}$. It is observed that the QP weight is greater for the ferromagnetic than for the AF contribution. Furthermore, when both magnetic bands are considered, the QP weight resembles that of the AF character. This can be seen as a consequence of the different momentum dependence of the interaction vertices $M^{+}, M^{-}$. For all $\mathbf{k}$ and $\mathbf{q}$ $\sim 0$, the vertex $M_{\mathbf{q}, \mathbf{k}}^{-} \sim \sqrt{q}$, while $M_{\mathbf{q}, \mathbf{k}}^{+} \sim$ const $+q$. As stated 


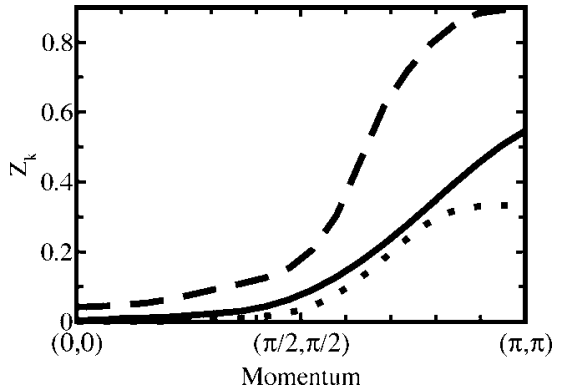

FIG. 5. QP weight along $(0,0) \rightarrow(\pi, \pi)$ for $J / t=0.4$ and $\theta$ $=40^{\circ}$. Contribution from the ferromagnetic band (dashed line), AF band (dotted), and both (solid line) in the SCBA.

before, since the QP excitation has a magnon assisted character driven by the vertex interaction, a stronger coupling produces an enhancement of the QP weight. So that, the coupling of the hole with the ferromagnetic band is more coherent than with the AF one, and when both couplings are considered together the QP weight follows the less coherent AF contribution.

Now we discuss the $J / t$ dependence of the QP excitations. As $J / t$ increases, we have observed that the character of the QP excitations changes from a many body state resulting from the dynamical coherent coupling of the hole with the magnons, to a free hole state weakly perturbed by the magnons. This crossover can be seen in more detail analyzing the QP wave function $\left|\Psi_{\mathbf{k}}\right\rangle{ }^{13,19,20}$ In general, $\left|\Psi_{\mathbf{k}}\right\rangle$ can be expressed as a sum of terms with one hole and different number of magnons, that in our case can be written as

$$
\left|\Psi_{\mathbf{k}}\right\rangle=a_{\mathbf{k}}^{(0)} h_{\mathbf{k}}^{\dagger}|\mathrm{AF}\rangle+\sum_{\mathbf{q}_{\mathbf{1}}, \sigma} a_{\mathbf{k}, \mathbf{q}_{1}}^{(1 \sigma)} h_{\mathbf{k}-\mathbf{q}_{1}}^{\dagger} \xi_{\mathbf{q}_{\mathbf{1}}}^{(\sigma) \dagger}|\mathrm{AF}\rangle+\cdots,
$$

where $\sigma= \pm$, and $\xi^{(\sigma)}$ represents the Bogoliubov operators $\xi^{(+)}=\eta$ and $\xi^{(-)}=\nu$. As $J / t$ increases, the multimagnon processes are energetically more expensive and their contributions to the QP wave function are notably reduced, whereas the zero and the one magnon terms become the relevant ones. ${ }^{13}$ Within the SCBA (Ref. 20) the one magnon coefficient is $a_{\mathbf{k}, \mathbf{q}_{1}}^{(1 \sigma)}=z_{\mathbf{k}} M_{\mathbf{k}, \mathbf{q}_{1}}^{\sigma} G_{\mathbf{k}-\mathbf{q}_{1}}^{h}\left(E_{\mathbf{k}}-\omega_{\mathbf{q}_{1}}^{\sigma}\right)$, while the zero magnon coefficient is $a_{\mathbf{k}}^{(0)}=z_{\mathbf{k}}$. The many-body state character of the QP excitation is signaled by the dependence of $a^{(1 \sigma)}$ with the hole Green function, which carries the information of the dynamical coupling of the hole with the magnons. In the weak coupling regime, large values of $J / t$, as $z_{\mathbf{k}} \rightarrow 1$ and $E_{\mathbf{k}} \rightarrow \epsilon_{\mathbf{k}}$ (see below), $a^{(1 \sigma)}$ becomes the first-order coefficient of a conventional Rayleigh-Schrödinger perturbation theory, $a_{\mathbf{k} \cdot \mathbf{q}}^{(1 \sigma)}=M_{\mathbf{k}, \mathbf{q}}^{\sigma} /\left(\epsilon_{\mathbf{k}}-\epsilon_{\mathbf{k}-\mathbf{q}}-\omega_{\mathbf{q}}^{\sigma}\right)$. In this coupling regime, the character of the QP wave function is that of a free hole state weakly renormalized by the one magnon excitations. This state correspond to the above mentioned $t$ resonance.

In Fig. 6 we show the QP, the $t$ resonance, and the bare hole energy dispersions for a canting angle $\theta=50^{\circ}$, in the strong, $J / t=0.4$, and the weak, $J / t=3$ coupling regimes. On one hand, for strong coupling (top panel), the QP energies are well separated from the bare hole and the $t$-resonance energies, indicating the highly nonperturbative character of the hole motion assisted by the magnons. On the other hand,

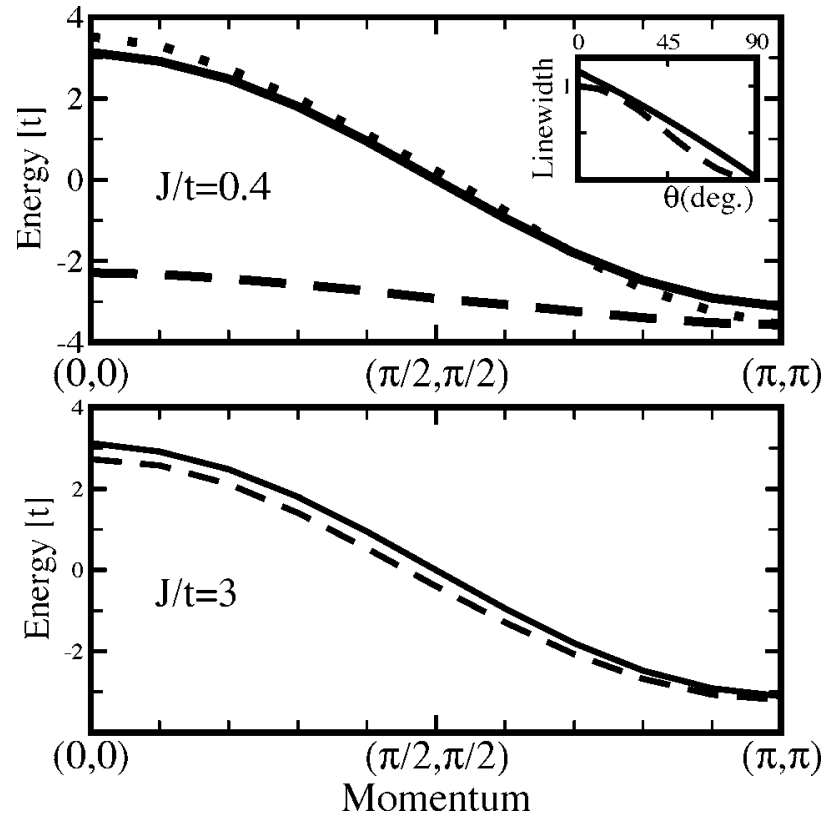

FIG. 6. Energy dispersion of the QP excitation (dashed line), bare hole excitation (solid line), and $t$ resonance excitation (dotted line) for an angle $\theta=50^{\circ}$. Top panel is for $J / t=0.4$. Inset: linewidth of the $t$ resonance (solid line) and $\cos ^{2} \theta$ (dashed line) as a function of $\theta$. Bottom panel is for $J / t=3$. For this regime the QP and the $t$-resonance excitations are the same.

once the crossover took place, for weak coupling (bottom panel), the QP and the $t$-resonance excitations have merged into a weakly perturbed state, whose dispersion closely follows the bare hole energy one. We have verified that the $t$-resonance dispersion is very well approximated by the weak coupling expression

$$
E_{\mathbf{k}}^{t}=\epsilon_{\mathrm{k}}+\sum_{\sigma \mathbf{q}} \frac{\left|M_{\mathbf{k}, \mathbf{q}}^{\sigma}\right|^{2}}{\epsilon_{\mathbf{k}}-\epsilon_{\mathrm{k}-\mathbf{q}}-\omega_{\mathbf{q}}^{\sigma}},
$$

in both, the strong and the weak coupling regimes. So, we can assure that the $t$ resonance can always be identified with the bare hole propagating along the ferromagnetic component, weakly perturbed by magnons. Another indication of the perturbative character of the $t$ resonance is the scaling of its linewidth with $M^{2} \sim \cos ^{2} \theta$ (see inset of Fig. 6). It is worth to stress that, while the decrease of the vertex interaction $M$ renders the $t$ resonance more coherent due to its perturbative character, it suppresses the coherence of the nonperturbative magnon assisted process. Finally, we have found that, in the weak coupling, the QP weight is well approximated by the expression $^{8}$

$$
z_{\mathbf{k}}=\frac{1}{1+\sum_{\mathbf{q} \sigma}\left(M_{\mathbf{k}, \mathbf{q}}^{\sigma} / \epsilon_{\mathbf{k}}-\epsilon_{\mathbf{k}-\mathbf{q}}-\omega_{\mathbf{q}}\right)^{2}} \rightarrow 1 .
$$

\section{Strings excitations}

In this section we analyze the dependence of the strings with the canting angle. Our general picture is based on Fig. 
TABLE I. Exponents $\alpha$ for the energy scaling $E \sim(J / t)^{\alpha}$ of the QP and the first string calculated for a lattice of $N=40 \times 40$ and several angles for $\mathbf{k}=(\pi / 2, \pi / 2)$. In the case of QP energies the parameter region is $0.01<J / t<0.3$ while for the strings 0.01 $<J / t<0.1$. To obtain better fits it has been considered the energy contribution of the magnetic background as in Ref. 7.

\begin{tabular}{ccccccc}
\hline \hline$\theta$ & $0^{\circ}$ & $10^{\circ}$ & $20^{\circ}$ & $30^{\circ}$ & $40^{\circ}$ & $50^{\circ}$ \\
\hline QP & 0.66 & 0.66 & 0.67 & 0.69 & 0.72 & 0.74 \\
String & 0.68 & 0.68 & 0.65 & 0.65 & 0.67 & 0.74 \\
\hline \hline
\end{tabular}

2. For $\theta=0^{\circ}$ there are several resonances above the QP peak which are interpreted as string excitations that result from the linear potential generated by paths of overturned spins in the $\mathrm{AF}$ background. This was confirmed in the $\mathrm{SCBA}^{3}$ by noting that their energies scale as $E_{\text {string }} \sim(J / t)^{\alpha}$, with $\alpha=2 / 3$. Notice that in the string picture the same exponent $2 / 3$ is obtained for the QP energy since the precursors of the quasiparticle excitation are just the strings. ${ }^{9}$ As the ferromagnetic channel of the underlying magnetic background is enhanced, as a consequence of greater canting angles, the AF channel is weakened and the string excitations start to smear out gradually until at angles around $\theta=40^{\circ}$ they disappear. This can be related to the fact that, at greater angles $\theta$, the AF channel has been reduced, so that the hole feels a weaker (sublinear) confining potential. To quantify this behavior we have computed the dependence of the QP and first string energies with $J / t$ by varying the canting angle, for a range of $0.01<J / t$ $<0.3$. In general, for all the momenta investigated, we have found values of the exponent $\alpha$ quite close to 2/3 for the QP and the first string excitation energies. As $\theta$ is increased the value of $\alpha$ remains approximately constant until for angles greater than $40^{\circ}$ there is a depart from $2 / 3$ to larger values. In Table I we display the dependence of the $\alpha$ exponent for several angles for the QP and the first string energies, at momentum $\mathbf{k}=(\pi / 2, \pi / 2)$. To provide a deeper insight of the type of potential implied by these energy exponents $\alpha$, it is useful to make a simple variational calculation of one particle within a one-dimensional potential. Let us assume a Hamiltonian $H=T+V$ where $T$ is the kinetic energy and the potential energy is of the type $V(x)=(J / t)|x|^{\beta}$. Then, if we propose a variational wave function for the particle of Gaussian form $\phi(x)=A \exp -\frac{K^{2} x^{2}}{2}$, with $K$ the variational parameter, it is straightforward to calculate the energy $E(K)=\frac{\langle\phi|H| \phi\rangle}{\langle\phi \mid \phi\rangle}$. Subsequent minimization with respect to $K$ leads to an energy dependence $E \sim(J / t)^{2 /(\beta+2)}$. For a linear potential it is recovered the expected value $\alpha=2 / 3$, whereas a sublinear potential, $\beta<1$, implies $\alpha>2 / 3$, in agreement with Table I.

\section{CONCLUSIONS}

We have made a detailed analysis of the competing mechanisms for hole motion in a canted antiferromagnetic background. To study the hole dynamics we have introduced the spinless fermion representation for the constrained fermi- ons of the $t-J$ model on a square lattice. We have modeled the canted antiferromagnetic background adding a Zeeman term to the $t-J$ model, whose effect is to tilt the Néel order giving rise to a ferromagnetic component. The problem thus formulated allows to study the hole dynamics continually from the pure antiferromagnetic case to the pure ferromagnetic one. As it is well known, in an unfrustrated Néel order a hole can only propagate by emitting and absorbing magnons, while for the pure ferromagnetic case the hole propagates freely. Here we have analyzed the evolution of the hole dynamics as a function of the canting angles by computing the hole spectral function. For this purpose we have use a reliable analytical method for the single hole case such as the self-consistent Born approximation.

We have found a complex momentum and canting angle dependence of the spectra. For $t>J$, the hole propagates preferably at two well separated energies: as a coherent spin polaron excitation at low energy, and as a quasifree hole at higher energy. In particular, from moderate to large canting angles, the quasiparticle spectral weight is considerably reduced, vanishing for momenta inside the magnetic Brillouin zone. This unexpected result is a consequence of the interference of the two mechanism for hole motion, originated from the antiferromagnetic and the ferromagnetic components of the underlying magnetic order. In the strong coupling regime $(t>J)$ the quasiparticle excitation has its origin in the AF component, namely, it is a many body state composed by a hole coherently coupled with the magnons. For this reason, when the canting angle increases, so AF component of the magnetic order is reduced, the QP weight goes to zero. Closely related to the QP excitations, we have found string excitations that rapidly smear out as the canting angle is increased. On the other hand, at higher energies we have found rather long-lived resonances related to the motion of the hole along the ferromagnetic component. As the ferromagnetic component increases with the canting angle, these resonances become more pronounced. Even in the strong coupling regime, we were able to fit the position and the linewidth of these resonances by a conventional perturbative calculation. As the system moves to the weak coupling regime we found that the character of QP excitations undergoes a crossover from the many-body spin polaron to a free hole state weakly perturbed by magnons.

We would like to emphasize that these features, obtained for the hole motion in a canted antiferromagnet, are generic and they could also be observed in other magnetic systems, where the anisotropies of the magnetic interactions lead to a canted magnetic state such as Dzyaloshinskii-Moriya or Ising anisotropies.

\section{ACKNOWLEDGMENTS}

The authors acknowledge the Brasil-Argentina Scientific Agreement through Project No. CAPES/SECYT 088/05. This work was also supported by the ANPCYT under Grant No. PICT2004 No 25724. I. J. H. thanks fundación J. Prats for partial support. 
${ }^{1}$ P. W. Anderson, Science 235, 1196 (1987).

${ }^{2}$ A. Damascelli, Z. Hussain, and Z.-X. Shen, Rev. Mod. Phys. 75, 473 (2003).

${ }^{3}$ Z. Liu and E. Manousakis, Phys. Rev. B 45, 2425 (1992).

${ }^{4}$ B. O. Wells, Z. -X. Shen, A. Matsuura, D. M. King, M. A. Kastner, M. Greven, and R. J. Birgeneau, Phys. Rev. Lett. 74, 964 (1995).

${ }^{5}$ R. B. Laughlin, Phys. Rev. Lett. 79, 1726 (1997).

${ }^{6}$ O. Rösch and O. Gunnarsson, Eur. Phys. J. B 43, 11 (2004); A. S. Mishchenko and N. Nagaosa, Phys. Rev. Lett. 93, 036402 (2004); O. Rösch, O. Gunnarsson, X. J. Zhou, T. Yoshida, T. Sasagawa, A. Fujimori, Z. Hussain, Z.-X. Shen, and S. Uchida, ibid. 95, 227002 (2005).

${ }^{7}$ G. Martinez and P. Horsch, Phys. Rev. B 44, 317 (1991).

${ }^{8}$ C. L. Kane, P. A. Lee, and N. Read, Phys. Rev. B 39, 6880 (1989).

${ }^{9}$ E. Dagotto, Rev. Mod. Phys. 66, 763 (1994).
${ }^{10}$ M. Brunner, F. F. Assaad, and A. Muramatsu, Phys. Rev. B 62, 15480 (2000).

${ }^{11}$ A. S. Mishchenko, N. V. Prokof'ev, and B. V. Svistunov, Phys. Rev. B 64, 033101 (2001).

${ }^{12}$ A. Lauchli and D. Poilblanc, Phys. Rev. Lett. 92, 236404 (2004).

${ }^{13}$ A. E. Trumper, C. J. Gazza, and L. O. Manuel, Phys. Rev. B 69, 184407 (2004); A. E. Trumper, C. J. Gazza, and L. O. Manuel, Physica B 354, 252 (2004).

${ }^{14}$ M. Vojta and K. W. Becker, Phys. Rev. B 57, 3099 (1998).

${ }^{15}$ F. Ronning, K. M. Shen, N. P. Armitage, A. Damascelli, D. H. Lu, Z.-X. Shen, L. L. Miller, and C. Kim, Phys. Rev. B 71, 094518 (2005).

${ }^{16}$ E. Manousakis, Rev. Mod. Phys. 63, 1 (1991).

${ }^{17}$ M. E. Zhitomirsky and T. Nikuni, Phys. Rev. B 57, 5013 (1998).

${ }^{18}$ Heinz Barentzen, J. Phys.: Condens. Matter 10, L699 (1998).

${ }^{19}$ G. F. Reiter, Phys. Rev. B 49, 1536 (1994).

${ }^{20}$ A. Ramsak and P. Horsch, Phys. Rev. B 57, 4308 (1998). 